\begin{tabular}{|c|l|}
\hline Title & Some Mathematical A spects of Quantum Zeno Effect \\
\hline Author(s) & A rai, A sao; Fuda, Toru \\
\hline Citation & $\begin{array}{l}\text { Letters in Mathematical Physics, 100/3),245-260 } \\
\text { https:/doi.org/L0.1007/311005-011-0539-0 }\end{array}$ \\
\hline Issue Date & 2012-06 \\
\hline Doc URL & http://hdl.handle.net/2115/52804 \\
\hline Rights & The original publication is available at www.springerlink.com \\
\hline Type & article (author version) \\
\hline File Information & LMP100-3_245-260.pdf \\
\hline
\end{tabular}

Instructions for use 


\title{
Some Mathematical Aspects of Quantum Zeno Effect
}

\author{
Asao Arai* and Toru Fuda \\ Department of Mathematics \\ Hokkaido University \\ Sapporo 060-0810 \\ Japan
}

\begin{abstract}
Mathematical investigations on quantum Zeno effect (QZE) are presented, including the following aspects: (i) QZE by frequent measurements made by an arbitrary partition of a time interval $[0, t](t>0)$; (ii) non-occurrence of QZE for vector states which are not in the domain of the Hamiltonian of the quantum system under consideration; (iii) asymptotic behavior of the survival probability characterizing QZE in the number $N$ of divisions of $[0, t]$; (iv) QZE along a curve in the Hilbert space of state vectors.
\end{abstract}

Mathematics Subject Classification (2010). 81Q10, 81P15, 47B25.

Keywords. quantum Zeno effect, survival probability, transition probability.

\section{Introduction}

The quantum phenomenon in which, by a series of measurements, transitions to states different from the initial state (the state at time zero) are hindered or inhibited, is called the quantum Zeno effect (QZE) [5]. It has been reported that the QZE can be experimentally realized (e.g., [1, 2, 3]).

In this paper, we are interested in investigating general mathematical aspects associated with the QZE. To explain new features in the present work, we first review the QZE briefly. So let us consider a quantum system $\mathrm{S}$ whose Hamiltonian is given by a self-adjoint operator $H$ on a complex Hilbert space $\mathcal{H}$ (so that $\mathcal{H}$ is a Hilbert space of state vectors of $\mathrm{S}$ ). We denote the inner product and the norm of $\mathcal{H}$ by $\langle\cdot, \cdot\rangle$ (anti-linear in the first variable and linear in the second) and $\|\cdot\|$, respectively. The domain of $H$ is denoted as $D(H)$. In what follows, we deal with vector states only. Hence we call a

\footnotetext{
${ }^{*}$ Corresponding author. E-mail: arai@math.sci.hokudai.ac.jp
} 
non-zero vector in $\mathcal{H}$ a state simply. As is well-known, by an axiom of quantum mechanics, for an initial state $\Psi \in \mathcal{H}$ with $\|\Psi\|=1$, the state at time $t \in \mathbb{R}$ is given by the vector $e^{-i t H} \Psi$, provided that no measurement is made in the time interval $[0, t]$, where $i$ is the imaginary unit and we use the physical unit system such that $\hbar:=h / 2 \pi$ ( $h$ is the Planck constant) is equal to 1 . Hence the probability of finding the initial state $\Psi$ by a measurement at time $t$ is given by $\left|\left\langle\Psi, e^{-i t H} \Psi\right\rangle\right|^{2}$. This quantity is called the survival probability of the initial state $\Psi$ at time $t$.

Now, consider a time interval $[0, t]$ with $t>0$ arbitrarily fixed and suppose that $N$ measurements, spaced equally in time by $t / N$, are made, where $N$ is an arbitrary natural number. If the state at time $j t / N(j=1, \cdots, N)$ is $\Phi \in \mathcal{H}$, then the state at time $(j+1) t / N$ is $e^{-i\{(j+1) t / N-j t / N\} H} \Phi=e^{-i t H / N} \Phi$, provided that no measurement is made in the time interval $[j t / N,(j+1) t / N]$. Hence the probability that, for all $j=1, \cdots, N$, the measurement at time $j t / N$ finds the initial state $\Psi$ is given by

$$
P_{N}(\Psi, t):=\left|\left\langle\Psi, e^{-i t H / N} \Psi\right\rangle\right|^{2 N}
$$

We call this quantity the multi-time survival probability of the initial state $\Psi$ in the time interval $[0, t]$.

We say that the QZE occurs with respect to the pair $(\Psi,[0, t])$ consisting of the initial state $\Psi$ and the time interval $[0, t]$ in the sense of equally spaced measurement in time if $\lim _{N \rightarrow \infty} P_{N}(\Psi, t)=1$. The occurrence of the QZE of this type physically means that, for all sufficiently large $N$, the successive measurements for the quantum system $\mathrm{S}$ at the times $t / N, 2 t / N, 3 t / N, \cdots, N t / N$ tend to maintain the initial state $\Psi$ with probability $\approx 1$, in other words, they tend to hinder transitions to states different from the initial state $\Psi$ with probability $\approx 1$.

Heuristically the occurrence of the QZE can be shown as follows (see, e.g., [4]). Using the formal expansion

$$
e^{-i t H}=I-i t H-\frac{t^{2}}{2} H^{2}+O\left(t^{3}\right) \quad(t \rightarrow 0),
$$

one infers that, for all $\Psi \in \cap_{n=1}^{\infty} D\left(H^{n}\right)$,

$$
\left|\left\langle\Psi, e^{-i s H} \Psi\right\rangle\right|^{2}=1-(\Delta H)_{\Psi}^{2} s^{2}+O\left(s^{4}\right) \quad(s \rightarrow 0),
$$

where

$$
(\Delta H)_{\Psi}:=\|(H-\langle\Psi, H \Psi\rangle) \Psi\|=\sqrt{\|H \Psi\|^{2}-\langle\Psi, H \Psi\rangle^{2}}
$$

is the uncertainty of $H$ in the state $\Psi$ (formula (1.2) can be easily made mathematically rigorous if $\Psi$ is an analytic vector of $H$ ). Hence, for all sufficiently large $N$,

$$
P_{N}(\Psi, t) \approx\left[1-(\Delta H)_{\Psi}^{2}\left(\frac{t}{N}\right)^{2}\right]^{N} \approx e^{-(\Delta H)_{\Psi}^{2} t^{2} / N} \approx 1 .
$$

In this way the occurrence of the QZE is inferred.

In the present paper, we begin with re-examining the QZE of the type described above in a mathematically rigorous and non-perturbative way in Section 2. We focus our attention on two aspects. One of them is to consider the situation where the $N$ measurements 
are made in a way not necessarily spaced equally in time. This generalization is mathematically natural. It may be physically meaningful too, because any measurement of the time inevitably has an error. We prove that, under such a situation too, a phenomenon regarded as a generalization of the QZE occurs, provided that the initial state is in $D(H)$ (Theorem 2.1). The other aspect is the possibility of non-occurrence of QZE for initial states not in $D(H)$, as suggested by the heuristic derivation of the QZE given above or the proof of Theorem 2.1 below. Indeed, there is an example in which the QZE does not occur for an initial state not in $D(H)$ (Example 2.4).

In the case of the QZE which occurs through frequent measurements spaced equally in time, it may be interesting to investigate the asymptotic expansion of the multi-time survival probability $P_{N}(\Psi, t)$ (see (1.1)) in the powers of $1 / N$ as $N \rightarrow \infty$. This is done in Section 3. For all $\Psi \in D(H)$ and $t>0$, we derive the asymptotic expansion of $P_{N}(\Psi, t)$ up to order $1 / N$.

In Section 4 , we consider measurements of states along a curve $\Psi(\cdot):[0, t] \rightarrow \mathcal{H}$, a strongly continuous mapping from $[0, t]$ to $\mathcal{H}$. This is a generalization of the situation considered in Section 2, because the constant mapping : $[0, t] \ni \lambda \mapsto \Psi(\lambda):=\Psi$ can be regarded as a special case of the curve. We prove that, for every partition $\left\{t_{0}, t_{1}, \cdots, t_{N}\right\}$ of $[0, t]$ with $0=t_{0}<t_{1}<\cdots<t_{N}=t$, the probability of finding the state $\Psi\left(t_{k}\right)$ at the time $t_{k}(k=1, \cdots, N)$ in the successive measurements at the times $t_{1}, \cdots, t_{N}$ tends to 1 as $N \rightarrow \infty$ (Theorem 4.2). Physically this means that very frequent measurements made successively along a curve prescribed in advance change the initial state $\Psi(0)$ to the final state $\Psi(t)$ with probability $\approx 1$.

In the last section, as an application of Theorem 4.2, we show that, for every pair $(\Psi, \Phi)$ of states in $\mathcal{H}$ with $\|\Psi\|=\|\Phi\|=1$, there exists a curve in $\mathcal{H}$ connecting $\Psi$ and $\Phi$ such that, through very frequent measurements at successive times given by a partition of the curve, $\Psi$ can be transformed to $\Phi$ with probability $\approx 1$. This is a refined version (in a sense) of von Neumann's discussion on a possible transformation, induced by frequent measurements, between arbitrary two states [6, Chapter 5], although the present case is restricted to vector states.

\section{QZE for an Arbitrary Partition of Time Interval}

Let $\Delta: t_{0}, t_{1}, \cdots, t_{N}\left(t_{j} \in[0, t], j=0, \cdots, N\right)$ be an arbitrary partition of the interval $[0, t]$ :

$$
0=t_{0}<t_{1}<\cdots<t_{N-1}<t_{N}=t
$$

We set

$$
\Delta_{k}:=t_{k}-t_{k-1}, \quad(k=1, \cdots, N), \quad|\Delta|:=\max _{1 \leq k \leq N} \Delta_{k} .
$$

Let $H$ be a self-adjoint operator on $\mathcal{H}$. Then, for each unit vector $\Psi \in \mathcal{H}$, we define a number

$$
P_{\Delta}(\Psi, t):=\prod_{k=1}^{N}\left|\left\langle\Psi, e^{-i \Delta_{k} H} \Psi\right\rangle\right|^{2} .
$$

In the context of quantum mechanics where $H$ represents the Hamiltonian of a quantum system, $P_{\Delta}(\Psi, t)$ is interpreted as the probability that, in the successive measurements at 
time $t_{1}, \cdots, t_{N}$ (measurements not necessarily spaced equally in time), the initial state $\Psi$ is found.

Theorem 2.1 For all $\Psi \in D(H)$ with $\|\Psi\|=1$,

$$
\lim _{|\Delta| \rightarrow 0} P_{\Delta}(\Psi, t)=1
$$

To prove this theorem, we need two lemmas.

\section{Lemma 2.2}

$$
\lim _{|\Delta| \rightarrow 0} \sum_{k=1}^{N} \Delta_{k}^{2}=0 .
$$

Proof. By direct computations, we have

$$
\sum_{k=1}^{N} \Delta_{k}^{2}=t^{2}-2 S_{\Delta}
$$

with

$$
S_{\Delta}:=t_{1}\left(t_{2}-t_{1}\right)+t_{2}\left(t_{3}-t_{2}\right)+\cdots+t_{N-1}\left(t_{N}-t_{N-1}\right) .
$$

Note that

$$
\lim _{|\Delta| \rightarrow 0} S_{\Delta}=\int_{0}^{t} x d x=\frac{t^{2}}{2}
$$

Hence (2.2) follows.

Lemma 2.3 For each $s \in \mathbb{R}$ and all $\Psi \in D(H)$ with $\|\Psi\|=1$,

$$
\left|\left\langle\Psi, e^{-i s H} \Psi\right\rangle\right|^{2} \geq 1-s^{2}\|H \Psi\|^{2} .
$$

Proof. Putting

$$
\alpha:=\left\langle\Psi,\left(e^{-i s H}-1\right) \Psi\right\rangle,
$$

we have

$$
\left\langle\Psi, e^{-i s H} \Psi\right\rangle=1+\alpha
$$

Hence

$$
\begin{aligned}
\left|\left\langle\Psi, e^{-i s H} \Psi\right\rangle\right|^{2} & \geq 1+\alpha+\alpha^{*} \\
& =1+\left\langle\Psi,\left(e^{-i s H}+e^{i s H}-2\right) \Psi\right\rangle \\
& =1-2 \beta
\end{aligned}
$$

with

$$
\beta:=\langle\Psi,(1-\cos (s H)) \Psi\rangle=\int_{\mathbb{R}}(1-\cos (s \lambda)) d\left\|E_{H}(\lambda) \Psi\right\|^{2},
$$

where $E_{H}(\cdot)$ is the spectral measure of $H$. One has

$$
0 \leq 1-\cos x \leq \frac{x^{2}}{2}, \quad \forall x \in \mathbb{R} .
$$

Hence

$$
0 \leq \beta \leq \frac{s^{2}}{2} \int_{\mathbb{R}} \lambda^{2} d\left\|E_{H}(\lambda) \Psi\right\|^{2}=\frac{s^{2}}{2}\|H \Psi\|^{2} .
$$

Thus (2.3) follows. 


\section{Proof of Theorem 2.1}

By the Schwarz inequality and the unitarity of $e^{-i s H}(\forall s \in \mathbb{R})$, we have $\left|\left\langle\Psi, e^{-i \Delta_{k} H} \Psi\right\rangle\right| \leq$

1. Hence $P_{\Delta}(\Psi, t) \leq 1$, which implies that

$$
\limsup _{|\Delta| \rightarrow 0} P_{\Delta}(\Psi, t) \leq 1
$$

By Lemma 2.3, we have

$$
\left|\left\langle\Psi, e^{-i \Delta_{k} H} \Psi\right\rangle\right|^{2} \geq 1-\Delta_{k}^{2}\|H \Psi\|^{2}
$$

For each $a>1$, we have

$$
1-x \geq e^{-a x}, \quad \forall x \in\left[0, \frac{\log a}{a}\right]
$$

Hence, taking $|\Delta|$ such that $|\Delta|^{2}\|H \Psi\|^{2} \leq \log a / a$, we have

$$
P_{\Delta}(\Psi, t) \geq e^{-a \sum_{k=1}^{N} \Delta_{k}^{2}\|H \Psi\|^{2}} .
$$

By this estimate and Lemma 2.2, we obtain

$$
\liminf _{|\Delta| \rightarrow 0} P_{\Delta}(\Psi, t) \geq 1
$$

which, combined with (2.7), gives (2.1).

We remark that the condition $\Psi \in D(H)$ in Theorem 2.1 is optimal. A counter example is given as follows.

Example 2.4 We consider the case where $\mathcal{H}=L^{2}(\mathbb{R})$ and $H$ is the Hamiltonian $H_{0}$ of a free quantum particle with mass $m>0$ moving in the one-dimensional space $\mathbb{R}$, i.e., $H_{0}:=p^{2} / 2 m, p:=-i D_{x}$ with $D_{x}$ being the generalized differential operator on $L^{2}(\mathbb{R})$ (in the variable $x \in \mathbb{R})$. Let $c>0$ be a constant and $\psi_{0} \in L^{2}(\mathbb{R})$ be such that its $L^{2}$-Fourier transform $\hat{\psi}_{0}$ takes the form

$$
\hat{\psi}_{0}(k)=\sqrt{\frac{2 c}{\pi}} \sqrt{\frac{|k|}{k^{4}+c^{2}}}, \quad k \in \mathbb{R} .
$$

It is easy to see that $\left\|\psi_{0}\right\|=1$ and $\psi_{0} \notin D\left(H_{0}\right)$. Moreover, we have for all $s \in \mathbb{R}$

$$
\left\langle\psi_{0}, e^{-i s H_{0}} \psi_{0}\right\rangle=\frac{4 c}{\pi} \int_{0}^{\infty} \frac{k e^{-i s k^{2} / 2 m}}{k^{4}+c^{2}} d k=e^{-|s| c / 2 m} .
$$

Hence, for all $t>0$

$$
\prod_{k=1}^{N}\left|\left\langle\psi_{0}, e^{-i \Delta_{k} H_{0}} \psi_{0}\right\rangle\right|^{2}=e^{-t c / m}
$$

Therefore

$$
\lim _{|\Delta| \rightarrow 0} \prod_{k=1}^{N}\left|\left\langle\psi_{0}, e^{-i \Delta_{k} H_{0}} \psi_{0}\right\rangle\right|^{2}=e^{-t c / m}<1 .
$$


Thus, in this case, Theorem 2.1 does not hold, physically meaning that the quantum Zeno effect does not occur. We also note that, for every $\varepsilon>0$,

$$
\lim _{|\Delta| \rightarrow 0} \prod_{k=1}^{N}\left|\left\langle\psi_{0}, e^{-i \Delta_{k} H_{0}} \psi_{0}\right\rangle\right|^{2}<\varepsilon
$$

if $c>-(m / t) \log \varepsilon$.

\section{Asymptotics of the Multi-time Survival Probabil- ity $P_{N}(\Psi, t)$ as $N \rightarrow \infty$}

In the case of the QZE caused by frequent measurements spaced equally in time, the asymptotic behavior (in $1 / N$ ) of the multi-time survival probability $P_{N}(\Psi, t)$ as $N \rightarrow \infty$ is interesting. It may be natural to infer that the asymptotic expansion of $P_{N}(\Psi, t)$ in $1 / N$ has the following form:

$$
P_{N}(\Psi, t)=1+c_{1}(\Psi, t) \frac{1}{N}+c_{2}(\Psi, t) \frac{1}{N^{2}}+\cdots \quad(N \rightarrow \infty)
$$

with $c_{n}(\Psi, t) \in \mathbb{R}(n=1,2, \cdots)$ being constants independent of $N$, expecting that each $c_{n}(\Psi, t)$ may have a physical meaning. In this section, we are concerned with this aspect and prove the following result:

Theorem 3.1 Let $t>0$. Then, for all $\Psi \in D(H)$ with $\|\Psi\|=1$,

$$
P_{N}(\Psi, t)=1-t^{2}(\Delta H)_{\Psi}^{2} \frac{1}{N}+o\left(\frac{1}{N}\right), \quad(N \rightarrow \infty) .
$$

Remark 3.2 The asymptotic formula (3.2) is only up to the first order $1 / N$. But we conjecture that it is possible to find higher order asymptotics in $1 / N$. We consider this aspect in a separate paper.

To prove Theorem 3.1, we need two lemmas:

Lemma 3.3 For all $s \in \mathbb{R}$ and $\Psi \in D(H)$,

$$
\begin{aligned}
\lim _{N \rightarrow \infty} N^{2}\left\langle\Psi,\left(1-\cos \frac{s H}{N}\right) \Psi\right\rangle & =\frac{1}{2} s^{2}\|H \Psi\|^{2}, \\
\lim _{N \rightarrow \infty} N\left\langle\Psi, \sin \frac{s H}{N} \Psi\right\rangle & =s\langle\Psi, H \Psi\rangle .
\end{aligned}
$$

Proof. We have

$$
N^{2}\left\langle\Psi,\left(1-\cos \frac{s H}{N}\right) \Psi\right\rangle=\int_{\mathbb{R}} N^{2}\left(1-\cos \frac{s \lambda}{N}\right) d\left\langle\Psi, E_{H}(\lambda) \Psi\right\rangle .
$$


It is easy to see that

$$
\lim _{N \rightarrow \infty} N^{2}\left(1-\cos \frac{s \lambda}{N}\right)=\frac{1}{2} s^{2} \lambda^{2}
$$

By (2.5), we have

$$
0 \leq N^{2}\left(1-\cos \frac{s \lambda}{N}\right) \leq \frac{1}{2} s^{2} \lambda^{2} .
$$

By functional calculus, we have

$$
\int_{\mathbb{R}} \frac{1}{2} s^{2} \lambda^{2} d\left\langle\Psi, E_{H}(\lambda) \Psi\right\rangle=\frac{1}{2} s^{2}\|H \Psi\|^{2}<\infty .
$$

Hence, by the Lebesgue dominated convergence theorem, we obtain (3.3).

We next prove (3.4). We have

$$
N\left\langle\Psi, \sin \frac{s H}{N} \Psi\right\rangle=\int_{\mathbb{R}} N \sin \frac{s \lambda}{N} d\left\langle\Psi, E_{H}(\lambda) \Psi\right\rangle .
$$

By the elementary inequality $|\sin x| \leq|x|, \forall x \in \mathbb{R}$, we obtain

$$
\left|N \sin \frac{s \lambda}{N}\right| \leq|s \lambda|
$$

By the Schwarz inequality, we have

$$
\int_{\mathbb{R}}|\lambda| d\left\langle\Psi, E_{H}(\lambda) \Psi\right\rangle \leq\|\Psi\|\left(\int_{\mathbb{R}} \lambda^{2} d\left\langle\Psi, E_{H}(\lambda) \Psi\right\rangle\right)^{\frac{1}{2}}=\|\Psi\| \cdot\|H \Psi\|<\infty .
$$

Moreover,

$$
\lim _{n \rightarrow \infty} N \sin \frac{s \lambda}{N}=s \lambda .
$$

Thus, by the Lebesgue dominated convergence theorem, we obtain (3.4).

Lemma 3.4 For all $n \in \mathbb{N}$,

$$
\begin{aligned}
& (1-x)^{n} \geq 1-n x, \quad 0 \leq \forall x \leq 1 \\
& (1-x)^{n} \leq 1-n x+(n x)^{2} e^{n x}, \quad \forall x \geq 0 .
\end{aligned}
$$

Proof. Inequality (3.5) is elementary. As for (3.6), we proceed as follows: For all $x \geq 0$ and $n \geq 2$,

$$
\begin{aligned}
(1-x)^{n} & =1-n x+\sum_{k=2}^{n} \frac{n(n-1) \cdots(n-k+1)}{k !}(-1)^{k} x^{k} \\
& \leq 1-n x+\sum_{k=2}^{n} \frac{n^{k}}{k !} x^{k} \leq 1-n x+(n x)^{2} \sum_{k=0}^{n-2} \frac{(n x)^{k}}{k !} \\
& \leq 1-n x+(n x)^{2} e^{n x} .
\end{aligned}
$$

Thus (3.6) holds. 


\section{Proof of Theorem 3.1}

Let

$$
a_{N}:=\left\langle\Psi,\left(1-\cos \frac{t H}{N}\right) \Psi\right\rangle, \quad b_{N}:=\left\langle\Psi, \sin \frac{t H}{N} \Psi\right\rangle .
$$

Then $a_{N}, b_{N} \in \mathbb{R}$ and $\left\langle\Psi, e^{-i \frac{t}{N} H} \Psi\right\rangle=1-a_{N}-i b_{N}$. Hence

$$
\left|\left\langle\Psi, e^{-i \frac{t}{N} H} \Psi\right\rangle\right|^{2}=\left(1-a_{N}\right)^{2}+b_{N}^{2}=1-\frac{q_{N}}{N^{2}},
$$

where

$$
q_{N}:=2 N^{2} a_{N}-\left(N b_{N}\right)^{2}-\frac{\left(N^{2} a_{N}\right)^{2}}{N^{2}}
$$

Therefore we have

$$
P_{N}(\Psi, t)=\left(1-\frac{q_{N}}{N^{2}}\right)^{N}
$$

By Lemma 3.3, we have

$$
\lim _{N \rightarrow \infty} N^{2} a_{N}=\frac{1}{2} t^{2}\|H \Psi\|^{2}, \quad \lim _{N \rightarrow \infty} N b_{N}=t\langle\Psi, H \Psi\rangle .
$$

Hence

$$
\lim _{N \rightarrow \infty} q_{N}=t^{2}\|H \Psi\|^{2}-t^{2}|\langle\Psi, H \Psi\rangle|^{2}=t^{2}(\Delta H)_{\Psi}^{2} .
$$

Moreover, it follows from Lemma 3.4 that, if $q_{N} \leq N$, then

$$
1-\frac{q_{N}}{N} \leq\left(1-\frac{q_{N}}{N^{2}}\right)^{N} \leq 1-\frac{q_{N}}{N}+\left(\frac{q_{N}}{N}\right)^{2} e^{\frac{q_{N}}{N}}
$$

which implies that

$$
q_{N}-\frac{q_{N}^{2}}{N} e^{\frac{q_{N}}{N}} \leq N\left(1-P_{N}(\Psi, t)\right) \leq q_{N}
$$

Hence

$$
\lim _{N \rightarrow \infty} N\left(1-P_{N}(\Psi, t)\right)=\lim _{N \rightarrow \infty} q_{N}=t^{2}(\Delta H)_{\Psi}^{2}
$$

Putting

$$
c_{N}:=t^{2}(\Delta H)_{\Psi}^{2} \frac{1}{N}-\left(1-P_{N}(\Psi, t)\right)
$$

we have

$$
P_{N}(\Psi, t)=1-t^{2}(\Delta H)_{\Psi}^{2} \frac{1}{N}+c_{N}
$$

By (3.7), we have $\lim _{N \rightarrow \infty} N c_{N}=0$, which means that $c_{N}=o(1 / N)(N \rightarrow \infty)$. Thus (3.2) holds. 


\section{General Mathematical Structure Behind QZE}

In this section, as a generalization of the QZE considered in Section 2, we consider the physical situation where measurements for states are made along a curve in the Hilbert space $\mathcal{H}$.

Let $\Psi(\cdot):[0, t] \rightarrow \mathcal{H}$ (a mapping from $[0, t]$ to $\mathcal{H}$ ) such that $\|\Psi(\lambda)\|=1, \forall \lambda \in[0, t]$ and consider

$$
P_{\Delta}(\Psi(\cdot), t):=\prod_{k=1}^{N}\left|\left\langle\Psi\left(t_{k}\right), e^{-i \Delta_{k} H} \Psi\left(t_{k-1}\right)\right\rangle\right|^{2} .
$$

This quantity is physically interpreted as the probability that, in the successive measurement at time $t_{1}, \cdots, t_{N}$, the state $\Psi\left(t_{k}\right)$ is found at time $t_{k}(k=1, \cdots, N)$.

Remark 4.1 For a unit vector $\Psi \in \mathcal{H}$, one can consider a constant mapping $\Psi_{\text {const }}(\cdot)$ : $[0, t] \rightarrow \mathcal{H}$ defined by $\Psi_{\text {const }}(\lambda):=\Psi, \forall \lambda \in[0, t]$. In this case, we have $P_{\Delta}\left(\Psi_{\text {const }}(\cdot), t\right)=$ $P_{\Delta}(\Psi, t)$, i.e., the case considered in Section 2. Thus $P_{\Delta}(\Psi(\cdot), t)$ is a generalization of $P_{\Delta}(\Psi, t)$.

Theorem 4.2 Let $\Psi(\cdot):[0, t] \rightarrow \mathcal{H}$ such that, for all $\lambda \in[0, t], \Psi(\lambda) \in D(H)$ and $\|\Psi(\lambda)\|=1$. Assume the following:

$$
\begin{aligned}
& \xi:=\sup _{0 \leq \lambda \leq t}\|H \Psi(\lambda)\|<\infty, \\
& \eta:=\sup _{\substack{\lambda, \nu \in[0, t] \\
\lambda \neq \nu}} \frac{\|\Psi(\lambda)-\Psi(\nu)\|}{|\lambda-\nu|}<\infty, \\
& \lim _{|\Delta| \rightarrow 0} \sum_{k=1}^{N} \operatorname{Re}\left\langle\Psi\left(t_{k}\right)-\Psi\left(t_{k-1}\right), \Psi\left(t_{k-1}\right)\right\rangle=0,
\end{aligned}
$$

where, for a complex number $z$, Re $z$ denotes its real part. Then

$$
\lim _{|\Delta| \rightarrow 0} P_{\Delta}(\Psi(\cdot), t)=1
$$

Remark 4.3 Condition (4.3) implies that $\|\Psi(\lambda)-\Psi(\nu)\| \leq \eta|\lambda-\mu|, \forall \lambda, \mu \in[0, t]$ (Lipschitz continuity). In particular, $\Psi(\cdot)$ is strongly continuous, so that the mapping $\Psi(\cdot):[0, t] \rightarrow \mathcal{H}$ is a curve in $\mathcal{H}$.

Proof. By the Schwarz inequality and the unitarity of $e^{-i s H}(\forall s \in \mathbb{R})$, we have

$$
\left|\left\langle\Psi\left(t_{k}\right), e^{-i \Delta_{k} H} \Psi\left(t_{k-1}\right)\right\rangle\right|^{2} \leq 1 .
$$

Hence

$$
P_{\Delta}(\Psi(\cdot), t) \leq 1
$$

For $k=1, \cdots, N$, we set

$$
\begin{aligned}
& a_{k}:=\left\langle\Psi\left(t_{k-1}\right),\left(e^{-i \Delta_{k} H}-1\right) \Psi\left(t_{k-1}\right)\right\rangle, \\
& b_{k}:=\left\langle\Psi\left(t_{k}\right)-\Psi\left(t_{k-1}\right),\left(e^{-i \Delta_{k} H}-1\right) \Psi\left(t_{k-1}\right)\right\rangle, \\
& c_{k}:=\left\langle\Psi\left(t_{k}\right)-\Psi\left(t_{k-1}\right), \Psi\left(t_{k-1}\right)\right\rangle .
\end{aligned}
$$


Then we have

$$
\begin{aligned}
\left|\left\langle\Psi\left(t_{k}\right), e^{-i \Delta_{k} H} \Psi\left(t_{k-1}\right)\right\rangle\right|^{2} & =\left|1+a_{k}+b_{k}+c_{k}\right|^{2} \\
& \geq 1+2 \operatorname{Re} a_{k}+2 \operatorname{Re} b_{k}+2 \operatorname{Re} c_{k} .
\end{aligned}
$$

By (2.4), (2.6) and (4.2), we have

$$
1+2 \operatorname{Re} a_{k} \geq 1-\Delta_{k}^{2}\left\|H \Psi\left(t_{k-1}\right)\right\|^{2} \geq 1-\Delta_{k}^{2} \xi^{2}
$$

By the Schwarz inequality, we have

$$
\left|\operatorname{Re} b_{k}\right| \leq\left|b_{k}\right| \leq\left\|\Psi\left(t_{k}\right)-\Psi\left(t_{k-1}\right)\right\| \cdot\left\|\left(e^{-i \Delta_{k} H}-1\right) \Psi\left(t_{k-1}\right)\right\| .
$$

Assumption (4.3) implies that

$$
\left\|\Psi\left(t_{k}\right)-\Psi\left(t_{k-1}\right)\right\| \leq \Delta_{k} \eta
$$

On the other hand, we have

$$
\left\|\left(e^{-i \Delta_{k} H}-1\right) \Psi\left(t_{k-1}\right)\right\|^{2}=\int_{\mathbb{R}}\left|e^{-i \Delta_{k} \lambda}-1\right|^{2} d\left\|E_{H}(\lambda) \Psi\left(t_{k-1}\right)\right\|^{2} .
$$

Using the elementary inequality

$$
\left|e^{-i x}-1\right|^{2} \leq x^{2}, \quad \forall x \in \mathbb{R},
$$

we obtain

$$
\begin{aligned}
\int_{\mathbb{R}}\left|e^{-i \Delta_{k} \lambda}-1\right|^{2} d\left\|E_{H}(\lambda) \Psi\left(t_{k-1}\right)\right\|^{2} & \leq \int_{\mathbb{R}} \Delta_{k}^{2} \lambda^{2} d\left\|E_{H}(\lambda) \Psi\left(t_{k-1}\right)\right\|^{2} \\
& =\Delta_{k}^{2}\left\|H \Psi\left(t_{k-1}\right)\right\|^{2} .
\end{aligned}
$$

Hence

$$
\left\|\left(e^{-i \Delta_{k} H}-1\right) \Psi\left(t_{k-1}\right)\right\| \leq \Delta_{k}\left\|H \Psi\left(t_{k-1}\right)\right\| \leq \Delta_{k} \xi
$$

Therefore

$$
\left|\operatorname{Re} b_{k}\right| \leq \xi \eta \Delta_{k}^{2}
$$

Thus we obtain

$$
2 \operatorname{Re} b_{k} \geq-2 \xi \eta \Delta_{k}^{2}
$$

By estimates (4.9) and (4.10), we have

$$
1+2 \operatorname{Re} a_{k}+2 \operatorname{Re} b_{k}+2 \operatorname{Re} c_{k} \geq 1-\left\{\left(\xi^{2}+2 \xi \eta\right) \Delta_{k}^{2}-2 \operatorname{Re} c_{k}\right\} .
$$

Note that, by (4.6) and (4.8), $1 \geq 1+2 \operatorname{Re} a_{k}+2 \operatorname{Re} b_{k}+2 \operatorname{Re} c_{k}$. Hence $\left(\xi^{2}+2 \xi \eta\right) \Delta_{k}^{2}-$ $2 \operatorname{Re} c_{k} \geq 0$. We also have

$$
\left|\operatorname{Re} c_{k}\right| \leq\left|c_{k}\right| \leq\left\|\Psi\left(t_{k}\right)-\Psi\left(t_{k-1}\right)\right\| \leq \eta \Delta_{k}
$$


Hence

$$
0 \leq\left(\xi^{2}+2 \xi \eta\right) \Delta_{k}^{2}-2 \operatorname{Re} c_{k} \leq\left(\xi^{2}+2 \xi \eta\right) \Delta_{k}^{2}+2 \eta \Delta_{k} \leq\left(\xi^{2}+2 \xi \eta\right)|\Delta|^{2}+2 \eta|\Delta| .
$$

Let $a>1$ be a constant and take $|\Delta|$ such that

$$
\left(\xi^{2}+2 \xi \eta\right)|\Delta|^{2}+2 \eta|\Delta| \leq \frac{\log a}{a} .
$$

Then, by (4.11), we have for $k=1, \cdots, N$

$$
0 \leq\left(\xi^{2}+2 \xi \eta\right) \Delta_{k}^{2}-2 \operatorname{Re} c_{k} \leq \frac{\log a}{a}
$$

Hence, by (2.8), we obtain

$$
1-\left\{\left(\xi^{2}+2 \xi \eta\right) \Delta_{k}^{2}-2 \operatorname{Re} c_{k}\right\} \geq \exp \left[-a\left\{\left(\xi^{2}+2 \xi \eta\right) \Delta_{k}^{2}-2 \operatorname{Re} c_{k}\right\}\right] .
$$

Therefore

$$
\begin{aligned}
P_{\Delta}(\Psi(\cdot), t) & \geq \prod_{k=1}^{N} \exp \left[-a\left\{\left(\xi^{2}+2 \xi \eta\right) \Delta_{k}^{2}-2 \operatorname{Re} c_{k}\right\}\right] \\
& =\exp \left[-a\left\{\left(\xi^{2}+2 \xi \eta\right) \sum_{k=1}^{N} \Delta_{k}^{2}-2 \sum_{k=1}^{N} \operatorname{Re} c_{k}\right\}\right]
\end{aligned}
$$

By Lemma 2.2 and (4.4),

$$
\lim _{|\Delta| \rightarrow 0}\left\{\left(\xi^{2}+2 \xi \eta\right) \sum_{k=1}^{N} \Delta_{k}^{2}-2 \sum_{k=1}^{N} \operatorname{Re} c_{k}\right\}=0
$$

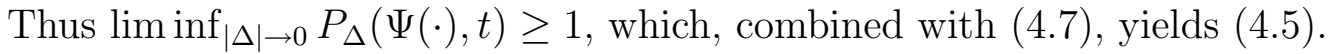

Corollary 4.4 Let $\Psi(\cdot):[0, t] \rightarrow \mathcal{H}$ be a strongly differentiable mapping from $[0, t]$ to $\mathcal{H}$ such that the following conditions hold:

(i) For all $\lambda \in[0, t], \Psi(\lambda) \in D(H)$ and $\|\Psi(\lambda)\|=1$.

(ii) (4.2) holds and

$$
\delta:=\sup _{0 \leq \lambda \leq t}\left\|\Psi^{\prime}(\lambda)\right\|<\infty,
$$

where $\Psi^{\prime}(\cdot)$ denotes the strong derivative of $\Psi(\cdot)$.

Then (4.5) holds.

Proof. By Theorem 4.2, it is sufficient to prove that (4.3) and (4.4) hold. By the strong differentiability, we have for all $\lambda, \nu \in[0, t]$

$$
\Psi(\lambda)-\Psi(\nu)=\int_{\nu}^{\lambda} \Psi^{\prime}(s) d s
$$


where the integral is taken in the sense of Bochner integral. Hence

$$
\|\Psi(\lambda)-\Psi(\nu)\| \leq\left|\int_{\nu}^{\lambda}\left\|\Psi^{\prime}(s)\right\| d s\right| \leq \delta|\lambda-\nu| .
$$

Thus (4.3) holds.

By (4.13), we have

$$
\Psi\left(t_{k}\right)-\Psi\left(t_{k-1}\right)=\int_{t_{k-1}}^{t_{k}} \Psi^{\prime}(\lambda) d \lambda, \quad k=1, \cdots, N .
$$

Let $\chi_{\left(t_{k-1}, t_{k}\right]}$ be the characteristic function of the interval $\left(t_{k-1}, t_{k}\right]$. Then

$$
\begin{aligned}
\sum_{k=1}^{N} \operatorname{Re}\left\langle\Psi\left(t_{k}\right)-\Psi\left(t_{k-1}\right), \Psi\left(t_{k-1}\right)\right\rangle & =\operatorname{Re} \sum_{k=1}^{N}\left\langle\int_{t_{k-1}}^{t_{k}} \Psi^{\prime}(\lambda) d \lambda, \Psi\left(t_{k-1}\right)\right\rangle \\
& =\operatorname{Re} \sum_{k=1}^{N} \int_{t_{k-1}}^{t_{k}}\left\langle\Psi^{\prime}(\lambda), \Psi\left(t_{k-1}\right)\right\rangle d \lambda \\
& =\operatorname{Re} \sum_{k=1}^{N} \int_{0}^{t} \chi_{\left(t_{k-1}, t_{k}\right]}(\lambda)\left\langle\Psi^{\prime}(\lambda), \Psi\left(t_{k-1}\right)\right\rangle d \lambda \\
& =\operatorname{Re} \int_{0}^{t}\left\langle\Psi^{\prime}(\lambda), \sum_{k=1}^{N} \chi_{\left(t_{k-1}, t_{k}\right]}(\lambda) \Psi\left(t_{k-1}\right)\right\rangle d \lambda
\end{aligned}
$$

For all $\lambda \in(0, t]$, we have

$$
\begin{array}{r}
\left|\left\langle\Psi^{\prime}(\lambda), \sum_{k=1}^{N} \chi_{\left(t_{k-1}, t_{k}\right]}(\lambda) \Psi\left(t_{k-1}\right)\right\rangle\right| \leq \delta \sum_{k=1}^{N} \chi_{\left(t_{k-1}, t_{k}\right]}(\lambda)=\delta, \\
\quad \lim _{|\Delta| \rightarrow 0}\left\langle\Psi^{\prime}(\lambda), \sum_{k=1}^{N} \chi_{\left(t_{k-1}, t_{k}\right]}(\lambda) \Psi\left(t_{k-1}\right)\right\rangle=\left\langle\Psi^{\prime}(\lambda), \Psi(\lambda)\right\rangle .
\end{array}
$$

Hence, by the Lebesgue dominated convergence theorem, we obtain

$$
\begin{aligned}
& \lim _{|\Delta| \rightarrow 0} \operatorname{Re} \int_{0}^{t}\left\langle\Psi^{\prime}(\lambda), \sum_{k=1}^{N} \chi_{\left(t_{k-1}, t_{k}\right]}(\lambda) \Psi\left(t_{k-1}\right)\right\rangle d \lambda \\
= & \operatorname{Re} \int_{0}^{t}\left\langle\Psi^{\prime}(\lambda), \Psi(\lambda)\right\rangle d \lambda=\frac{1}{2} \int_{0}^{t} \frac{d}{d \lambda}\|\Psi(\lambda)\|^{2} d \lambda \\
= & \frac{1}{2}\left(\|\Psi(t)\|^{2}-\|\Psi(0)\|^{2}\right)=0 .
\end{aligned}
$$

Thus (4.4) holds.

Example 4.5 Let $A$ be a self-adjoint operator on $\mathcal{H}$ and $\Psi_{0}$ be a vector in $\mathcal{H}$ satisfying the following conditions:

$$
\Psi_{0} \in D(A) \cap \bigcap_{0 \leq \lambda \leq t} D\left(H e^{-i \lambda A}\right), \quad \sup _{0 \leq \lambda \leq t}\left\|H e^{-i \lambda A} \Psi_{0}\right\|<\infty, \quad\left\|\Psi_{0}\right\|=1 .
$$


Then one can define a mapping $\Psi(\cdot):[0, t] \rightarrow \mathcal{H}$ by

$$
\Psi(\lambda):=e^{-i \lambda A} \Psi_{0}, \quad \lambda \in[0, t] .
$$

It is obvious that the mapping $\Psi(\cdot)$ satisfies condition (i) in Corollary 4.4. Moreover, $\Psi(\cdot)$ is strongly differentiable on $[0, t]$ and

$$
\left\|\Psi^{\prime}(\lambda)\right\|^{2}=\left\|A e^{-i \lambda A} \Psi_{0}\right\|^{2}=\left\|A \Psi_{0}\right\|^{2}
$$

so that $\Psi(\cdot)$ satisfies condition (ii) in Corollary 4.4 too. Thus, for this $\Psi(\cdot)$, (4.5) holds.

\section{Transition Between Arbitrary Two States by Mea- surements}

We fix two unit vectors $\Psi$ and $\Phi$ in $D(H)$ arbitrarily. Then one can define a strongly differentiable mapping $\Psi(\cdot):[0, t] \rightarrow \mathcal{H}$ connecting $\Psi$ and $\Phi$ as follows.

\section{(1) The case where $\Psi$ and $\Phi$ are linearly dependent}

In this case, there exists a constant $\alpha \in[0,2 \pi)$ such that $\Phi=e^{i \alpha} \Psi$. Then, defining $\Psi(\cdot):[0, t] \rightarrow \mathcal{H}$ by

$$
\Psi(\lambda):=e^{i \frac{\alpha \lambda}{t}} \Psi, \quad \lambda \in[0, t],
$$

we see that $\Psi(\cdot)$ is strongly differentiable on $[0, t]$ with $\Psi(0)=\Psi$ and $\Psi(t)=\Phi$.

(2) The case where $\Psi$ and $\Phi$ are linearly independent

In this case, let

$$
\Xi:=\frac{\Phi-\langle\Phi, \Psi\rangle \Psi}{\|\Phi-\langle\Phi, \Psi\rangle \Psi\|}
$$

Then $\{\Psi, \Xi\}$ is an orthonormal system in $\mathcal{H}$. It follows that there exist constants $\alpha, \beta, \gamma \in$ $[0,2 \pi)$ such that

$$
\Phi=(\cos \alpha) e^{i \beta} \Psi+(\sin \alpha) e^{i \gamma} \Xi .
$$

Using this fact, we define $\Psi(\cdot):[0, t] \rightarrow \mathcal{H}$ by

$$
\Psi(\lambda):=\left(\cos \frac{\alpha \lambda}{t}\right) e^{i \frac{\beta \lambda}{t}} \Psi+\left(\sin \frac{\alpha \lambda}{t}\right) e^{i \frac{\gamma \lambda}{t}} \Xi, \quad \lambda \in[0, t]
$$

It is easy to see that the mapping $\Psi(\cdot)$ is strongly differentiable on $[0, t]$ with $\Psi(0)=\Psi$ and $\Psi(t)=\Phi$.

Proposition 5.1 Let $\Psi(\cdot)$ be defined by (5.1) or (5.3). Then $\Psi(\cdot)$ satisfies all the assumptions of Corollary 4.4 with $\Psi(0)=\Psi$ and $\Psi(t)=\Phi$. 
Proof. We see from the definition of $\Psi(\cdot)$ that, for all $\lambda \in[0, t], \Psi(\lambda) \in D(H)$ and $\|\Psi(\lambda)\|=1$, and $\Psi(\cdot)$ is strongly differentiable on $[0, t]$ with $\Psi(0)=\Psi$ and $\Psi(t)=\Phi$.

We first consider the case where $\Psi$ and $\Phi$ are linearly dependent. In this case, we have

$$
\begin{aligned}
& \|H \Psi(\lambda)\|=\|H \Psi\|, \\
& \left\|\Psi^{\prime}(\lambda)\right\|=\frac{\alpha}{t} .
\end{aligned}
$$

Hence condition (ii) in Corollary 4.4 are satisfied.

Next, let $\Psi$ and $\Phi$ be linearly independent. Then

$$
\begin{aligned}
& \|H \Psi(\lambda)\| \leq\|H \Psi\|+\|H \Xi\|, \\
& \left\|\Psi^{\prime}(\lambda)\right\|=\left\|\left(-\frac{\alpha}{t} \sin \frac{\alpha \lambda}{t}+i \frac{\beta}{t} \cos \frac{\alpha \lambda}{t}\right) e^{i \frac{\beta \lambda}{t}} \Psi+\left(\frac{\alpha}{t} \cos \frac{\alpha \lambda}{t}+i \frac{\gamma}{t} \sin \frac{\alpha \lambda}{t}\right) e^{i \frac{\gamma \lambda}{t}} \Xi\right\| \\
& \leq \frac{2 \alpha+\beta+\gamma}{t} .
\end{aligned}
$$

Hence condition (ii) in Corollary 4.4 are satisfied.

Corollary 4.4 and Proposition 5.1 immediately lead one to the following fact:

Corollary 5.2 For the mapping $\Psi(\cdot)$ defined by (5.1) or (5.3), $\lim _{|\Delta| \rightarrow 0} P_{\Delta}(\Psi(\cdot), t)=1$.

Corollary 5.2 may be interpreted as follows: For every pair $(\Psi, \Phi)$ of states in $\mathcal{H}$ with $\|\Psi\|=\|\Phi\|=1$, there exists a curve in $\mathcal{H}$ connecting $\Psi$ and $\Phi$ such that, through very frequent measurements at successive times given by a partition of this curve, the state $\Psi$ can be transformed to $\Phi$ with probability $\approx 1$.

\section{Acknowledgments}

T. Fuda would like to thank S. Futakuchi, T. Muroi and M. Nagasaka for valuable comments. This work was supported by the Grant-In-Aid 21540206 for scientific research from Japan Society for the Promotion of Science (JSPS).

\section{References}

[1] O. Alter and Y. Yamamoto, Quantum Measurement of a Single System, John Wiley \& Sons, Inc., New York, 2001.

[2] D. Home and M. A. B. Whitaker, A Conceptual Analysis of Quantum Zeno; Paradox, Measurement, and Experiment, Annals of Physics 258(1997), 237-285.

[3] W. M. Itano, D. J. Heinzen, J. J. Bollinger, and D. J. Wineland, Quantum Zeno effect, Phys. Rev. A 41(1990), 2295.

[4] R. Joos, Decoherence Through Interaction with the Environment, Chapter 3, §3.3 in Decoherence and the Appearance of a Classical World in Quantum Theory (Editors: D. Giulini, E. Joos, C. Kiefer. J. Kupsch, I.-O. Stamatescu and H. D. Zeh), Springer, Berlin, Heidelberg, 1996. 
[5] B. Misra and E. C. G. Sudarshan, The Zeno's paradox in quantum theory, J. Math. Phys. 18 (1977), 756-763.

[6] J. von Neumann, Die Mathematische Grundlagen der Quantenmechanik, Springer, Berlin, 1932. Reprint:1981. 